\title{
Alterstice
}

Revue internationale de la recherche interculturelle

International Journal of Intercultural Research

Revista International de la Investigacion Intercultural

\section{La construction des espaces frontaliers européens : entre dynamisme et résistances}

\section{Jacqueline Breugnot}

Volume 2, numéro 1, 2012

Stratégies de (re)médiation en situation plurilingue. Études de cas en contextes de recherche et d'éducation

URI : https://id.erudit.org/iderudit/1077554ar

DOI : https://doi.org/10.7202/1077554ar

Aller au sommaire du numéro

Éditeur(s)

Alterstice

ISSN

1923-919X (numérique)

Découvrir la revue

Citer cet article

Breugnot, J. (2012). La construction des espaces frontaliers européens : entre dynamisme et résistances. Alterstice, 2(1), 67-77.

https://doi.org/10.7202/1077554ar

\section{Résumé de l'article}

Les zones frontalières européennes sont des espaces en mutation. Entre idéal d'identité européenne partagée et difficultés à coopérer au quotidien, les représentations de l'Autre, endormies pendant les décennies où la frontière était stable et protectrice, se trouvent remises en question. Les politiques, pour favoriser une porosité croissante de la frontière et pour répondre aux attentes des entreprises, ont développé des programmes d'apprentissage de la langue du voisin. Il semble qu'elles aient fait, là, abstraction des résistances, des différences culturelles et des handicaps de la proximité.

L'objectif de cette contribution est d'alimenter le débat scientifique autour de la construction des espaces frontaliers par des informations issues d'études menées sur différentes frontières européennes. Après une présentation des particularités de ces espaces, et plus particulièrement celles de l'espace du Rhin Supérieur, sont décrites les attitudes des populations face au défi des évolutions territoriales, entre militantisme, repli identitaire et indifférence. 


\section{간.}

alterstice

ARTICLE THÉMATIQUE

\section{La construction des espaces frontaliers européens : entre dynamisme et résistances}

Jacqueline Breugnot ${ }^{1}$

\section{Résumé}

Les zones frontalières européennes sont des espaces en mutation. Entre idéal d'identité européenne partagée et difficultés à coopérer au quotidien, les représentations de l'Autre, endormies pendant les décennies où la frontière était stable et protectrice, se trouvent remises en question. Les politiques, pour favoriser une porosité croissante de la frontière et pour répondre aux attentes des entreprises, ont développé des programmes d'apprentissage de la langue du voisin. Il semble qu'elles aient fait, là, abstraction des résistances, des différences culturelles et des handicaps de la proximité.

L'objectif de cette contribution est d'alimenter le débat scientifique autour de la construction des espaces frontaliers par des informations issues d'études menées sur différentes frontières européennes. Après une présentation des particularités de ces espaces, et plus particulièrement celles de l'espace du Rhin Supérieur, sont décrites les attitudes des populations face au défi des évolutions territoriales, entre militantisme, repli identitaire et indifférence.

\section{Rattachement de l'auteure}

${ }^{1}$ Institut für fremdsprachliche Philologien, Universität Koblenz-Landau, Landau, Allemagne

\section{Correspondance}

breugnot@uni-landau.de

\section{Mots clés}

espaces frontaliers; communication interculturelle

\section{Pour citer cet article :}

Breugnot, J. (2012). La construction des espaces frontaliers européens : entre dynamisme et résistances. Alterstice, 2(1), 67-78. 


\section{Introduction}

La construction européenne et la relative stabilisation politique des pays contributeurs ont permis d'imaginer l'émergence de nouvelles entités territoriales : les régions transfrontalières. Une diversité linguistique et culturelle y côtoie des mesures économiques et politiques, ainsi que des représentations symboliques et historiques renégociées.

Les politiques éducatives ${ }^{1}$ tentent de prendre en compte et de préparer les évolutions attendues; les efforts déployés se heurtent cependant à des résistances dont les causes et la mesure ont été souvent sous-estimées.

À partir de recherches menées dans 5 pays européens par des chercheurs de différentes disciplines², géopolitique, sociologie, psychanalyse, anthropologie et didactologie, l'article, après avoir clarifié la polysémie liée à la création de ces nouveaux espaces, tentera de mettre en lumière les politiques mises en œuvre et leurs implications pour les populations concernées.

\section{La notion de frontière dans les espaces géographiques pacifiés}

\section{Une polysémie}

Toute frontière assure une multiplicité de fonctions concernant divers registres, allant du privé, familial, à l'espace d'enjeux internationaux politiques et économiques. Le terme englobe des réalités disparates qui donnent lieu à des représentations également dissemblables. L'utilisation de plusieurs définitions partielles du terme frontière peut s'avérer pertinente à condition de restreindre leur application au niveau d'analyse dont elles sont issues. Seule l'articulation de toutes ces perspectives permet de comprendre la complexité des phénomènes frontaliers : en effet la frontière suppose une dimension politique mais elle est aussi un espace économique, où elle peut devenir un lieu de ressource. Elle reste cependant un lieu de vie, d'histoire et de mémoire. Elle peut comporter une dimension sociologique, sociale, culturelle, symbolique ou imaginaire (Odgers, 2001).

Du point de vue géopolitique, la frontière est la ligne ou la zone qui forme la limite d'un territoire, d'un État ou bien un ensemble politique que ses dirigeants cherchent à constituer en État plus ou moins indépendant. (Lacoste, 1993, p. 658)

Dans le cas des espaces frontaliers, ces nouvelles entités ne prévoient pas de se distinguer des territoires extérieurs (c'est-à-dire des territoires nationaux) dans un registre politique, mais les frontières intra-européennes nécessitent de faire appel à des catégories infra- et supranationales.

\section{Vers un idéal de l'effacement des frontières}

À la différence des frontières internes à un pays comme en Suisse ou au Canada où une pression politique pèse depuis longtemps pour maintenir une cohésion minimale et lutter contre les risques éventuels de séparatisme, la notion d'espace frontalier commun à deux ou trois pays est une notion récente. En fait, elle apparaît avec la construction européenne après la Seconde Guerre mondiale et se définit comme un nouvel espace en construction, destiné à affaiblir la fonction historique des frontières qui consistait à séparer officiellement deux populations se définissant par leur appartenance nationale, linguistique et culturelle. Le Pays basque fait toutefois exception, en ce qu'une partie de la population nie la séparation linguistique et culturelle.

Dans l'imaginaire d'une Europe harmonieuse, comme dans le réalisme d'accords économiques fructueux, la frontière est considérée comme mauvaise, a priori, car elle est supposée diviser. La représentation sous-entendue

\footnotetext{
${ }^{1}$ Que le lecteur, la lectrice, ne se laisse pas désorienter par l'évocation répétée de la question des mesures éducatives au cours de ce texte. Elle sera abordée selon différentes perspectives car elle est révélatrice aussi bien des représentations que des évolutions probables.

${ }^{2}$ Les références scientifiques qui fondent cette contribution proviennent en grande partie des coopérations mises en place à compter de 2005 dans le cadre de la préparation d'un projet de recherche Comenius. Voir Loyer (2006), Kurcz (2006), Berger (2007) et Bothorel-Witz (2000). Les échanges scientifiques et les coopérations perdurent avec la plupart d’entre eux.
} 
est que, sans les guerres ou les rivalités entre États, les populations cohabiteraient sans conflits dans un espace partagé (Loyer, 2006), ce qui témoigne pour le moins d'un bel optimisme, sinon d'une méconnaissance des concurrences locales.

À l'intérieur même de l'Europe, ces frontières présentaient, jusqu'à la chute du Mur de Berlin, un degré de porosité variable. La frontière entre l'Allemagne et l'Autriche était depuis longtemps poreuse alors que la frontière entre I'Allemagne et la Pologne restait encore quasi hermétique.

À partir de 1957, avec la création de la Communauté économique européenne et surtout depuis 1992 avec l'apparition de la notion de citoyenneté européenne, les instances politiques vont proposer de modifier le statut historique de la frontière et de construire de nouveaux espaces transnationaux destinés à se superposer aux États et aux nations.

Ces espaces prennent la forme d'Eurodistricts rassemblant au moins deux à trois villes, comme celui de StrasbourgKehl-Offenburg et pouvant aller jusqu'à 450 pour l'Eurodistrict catalan. Ils peuvent également prendre la forme de "Euregio » ou « Eurorégion » ou encore de " grande région ». Afin de renforcer leur identité symbolique, on a créé des noms tels que SAARLORLUX pour Saare/Lorraine/Luxembourg ou Rhin Supérieur pour un espace regroupant le canton de Bâle, une partie de l'Alsace, une bande frontalière du Bade-Wurtemberg et le sud du Palatinat, ou encore Viadrina ou Pomerania pour la région germano-polonaise.

Les réflexions à l'origine de ces nouvelles entités sont multiples et marquées par leur ancrage idéalo-politique. Pour certains, il s'agit d'une étape intermédiaire avant d'adopter une identité européenne supérieure à l'identité nationale. L'objectif est alors d'estomper peu à peu la force symbolique des frontières à l'intérieur de l'Europe. Pour d'autres, il s'agit également d'estomper les frontières mais avec l'objectif d'une Europe fédérale, reprenant plus ou moins le modèle allemand. La création d'identités territoriales pourvues de compétences spécifiques constituerait la structure de base. Ces compétences s'étendraient tant aux domaines économiques que politiques et éducatifs. La phase préparatoire consiste actuellement à affaiblir par petites touches les pouvoirs nationaux, en accordant, par exemple, les subventions européennes directement aux villes sans passer par les instances nationales ${ }^{3}$.

\section{Un pragmatisme de la nécessaire frontière}

Les prescriptions politiques s'appuient sur des affirmations dont la logique semble indiscutable: dans une Europe unie, il n'est plus pensable que des populations vivent à quelques kilomètres l'une de l'autre et persistent à se considérer comme parfaitement étrangères les unes aux autres. II semble plus aisé de se sentir européen, ce qui n'engage pas à grand-chose, que de partager une identité régionale commune avec ce que cela peut avoir de conséquences au quotidien.

Les besoins de différenciation culturelle de part et d'autre de la frontière persistent. Les affirmations qui en découlent reposent largement sur une méconnaissance de l'autre, méconnaissance accompagnée d'une conviction profondément ancrée du contraire.

\section{Les politiques mises en œuvre}

Afin de dynamiser ces nouveaux espaces, les responsables politiques, en accord avec les décideurs économiques ont pris différents types de mesures. La plupart de ces mesures ont été conçues au niveau du Parlement européen et beaucoup d'entre elles, même si elles peuvent différer dans leur transposition, sont comparables sur toutes les frontières européennes. Leurs financements ont des sources multiples et nécessitent souvent un outillage juridique complexe.

L'espace frontalier cité en référence est essentiellement l'espace du Rhin Supérieur, auquel s'ajoutent, à titre de comparaison, l'espace germano-polonais et l'espace franco-espagnol.

\footnotetext{
${ }^{3}$ Par exemple les programmes URBACT I et II.
} 


\section{Un large éventail d'interventions}

Les projets peuvent concerner le développement d'infrastructures (comme le port autonome de Szczecin, voir Kurcz, 2007), la création de zones industrielles indépendantes, la gestion commune de services (comme le jumelage de I'hôpital de Bayonne avec celui de Donastia-San Sebastián), l'aménagement commun du territoire (nouvelles voies de communication, extension du réseau de transports en commun, parcs binationaux comme entre Strasbourg et Kehl).

Les projets se sont développés de manière plus ou moins harmonieuse selon l'histoire qui a régi les relations des pays concernés. Ainsi, lorsque des accords existaient depuis longtemps, comme entre la France et l'Allemagne, les projets sont apparus et se sont développés peu à peu, alors que leur mise en place s'est montrée plus chaotique dans les espaces où l'ouverture de frontières autrefois hermétiques s'est réalisée brutalement, comme entre l'Allemagne et la Pologne.

Pour Kurcz (2007), l’arrivée de " partenaires » allemands associée à l'afflux de populations venues d'autres parties de la Pologne a bouleversé les infrastructures côté polonais. Les branches traditionnelles de l'industrie ont fait place à des activités nouvelles qui ont été source de conflit, par exemple entre les commerçants qui utilisaient les structures existantes et les nouveaux qui se sont spécialisés dans l'accueil des Allemands. Pour Kurcz, ces déséquilibres entre niveaux de rémunération, mais aussi entre perceptions de l'Histoire, devraient absolument être pris en compte dans la planification des coopérations transfrontalières.

Entre la France et l'Espagne, les coopérations transfrontalières existaient déjà il y a plusieurs siècles. Les Basques et les Béarnais ont longtemps vécu en voisins, sans pour autant penser que des liens supérieurs, nation basque ou fédération européenne, les unissaient au-delà d'un voisinage bien compris. Ce qui a changé, selon Loyer (2006), c'est la plus grande diversité des échanges, l'arsenal juridique qu'ils requièrent et surtout la volonté de créer un sentiment de communauté de destin, européen ou basque, entre les populations, d'où l'importance de lier l'analyse des réalisations à l'étude des représentations en présence.

Comme nous le voyons, la réalisation de tous ces projets, le développement économique de ces espaces nécessitent en amont une adaptation des populations concernées. Les mesures prises visent notamment à développer des compétences linguistiques appropriées. L'idéal serait de parvenir à un bilinguisme de fait dans toute la zone frontalière. Pour ce faire, se sont développées, quelquefois depuis la maternelle, des formes d'enseignement dans «la langue du voisin».

\section{L'enjeu des compétences linguistiques}

Le terme "langue du voisin » est utilisé dans les espaces frontaliers qui ne partagent pas de langue commune, tels les espaces germano-polonais. Dans les espaces franco-espagnols, ce sont davantage le catalan et le basque qui seront valorisés comme langue partagée, même si l’Euskara s'arrêtait anciennement au sud de Bayonne. Dans l'espace franco-allemand, l'expression "langue du voisin » a été utilisée des deux côtés de la frontière jusqu'en 2000, mais c'est finalement le terme de langue régionale qui a été retenu côté français, en définissant l'allemand standard comme forme écrite de l’alsacien. Pour Arlette Bothorel-Witz (2000),

La question des langues en Alsace suscite, au-delà des débats passionnés qui ressurgissent régulièrement, des visions contradictoires et non moins idéologiques (au sens propre du terme) dans la littérature spécialisée. Le simple repérage des langues en présence, leurs dénominations respectives font apparaître d'importantes divergences qui sont diversement fondées. Elles relèvent, pour l'essentiel, de l'appréciation très variable de la place de l'allemand dans la constellation actuelle, de ses rapports d'inclusion ou d'exclusion avec les dialectes alsaciens en synchronie et, parallèlement, de la polysémie (en fonction du cadre de leur emploi) des termes : « allemand » et « langue régionale ».

Au-delà des raisons idéologiques qui sous-tendent les représentations de la langue, le choix de la dénomination est important en ce qu'il déterminera les sources de financement et les libertés qui pourront être prises à l'égard du pouvoir national (voir par exemple Huck, 2005, sur l'évolution des manuels en Alsace). 


\section{L'exemple de la formation des enseignants}

La Conférence franco-germano-suisse du Rhin Supérieur, créée en 1991, a, entre autres, fondé la Confédération des Instituts universitaires de formation des maîtres du Rhin supérieur dont l'objectif est de " soutenir et développer les formations bilingues de professeurs des écoles dans l'espace du Rhin supérieur, de promouvoir les échanges des étudiants et des enseignants et d'œuvrer ensemble pour le bi- et plurilinguisme " ${ }^{4}$. C'est dans ce cadre qu'a été mis en place depuis 2006 un " Master Trinational Plurilinguisme » qui, " grâce à une formation adaptée et centrée sur une réalité linguistique frontalière omniprésente [...] fera des enseignants bilingues du Rhin Supérieur de demain, les garants d'un bilinguisme et d'une éducation interculturelle accessibles aux prochaines générations d'écoliers ${ }^{5}$. Ce projet a impliqué quelque huit universités, dont deux ont décidé entre-temps de se retirer devant le peu de succès rencontré auprès des étudiants.

La mise en place de tels projets fait bien apparaître la complexité des situations transfrontalières. En effet, les conditions de recrutement des enseignants, par exemple, sont différentes d'un pays à l'autre. La mise en place d'un master commun a demandé un engagement considérable de la part des universitaires impliqués pour parvenir à un consensus relatif aux contenus, mais le principe du recrutement sur concours en France et la nécessité en Allemagne de détenir un master labellisé " enseignement " enlève beaucoup d'attraits à ce master car pour une durée d'études équivalente, ceux qui auront suivi un cursus strictement national pourront prétendre à un statut de titulaire tandis que les étudiants du master trinational se contenteront d'un statut de vacataire. En outre, la question du salaire, même perçue ou présentée comme secondaire, peut s'avérer rapidement frustrante, puisqu'à formation identique et pour une charge de travail comparable dans des lieux géographiques très proches, les salaires varient presque du simple au double d'un côté à l'autre de la frontière.

Cette question des salaires interfère également dans les relations entre enseignants " nationaux » et " étrangers » lors des échanges d'enseignants. Les rectorats prévoient dans la plupart des espaces frontaliers européens la possibilité d'aller enseigner pendant trois ans voire plus dans le pays voisin, chacun gardant son salaire d'origine. Si ces écarts perturbent les relations dans le cas du Rhin Supérieur, ils se révèlent quasi rédhibitoires pour la réciprocité sur les frontières germano-tchèque ou germano-polonaise.

\section{L'exemple des projets scolaires}

Les actions menées par la Conférence du Rhin Supérieur concernent également les élèves du primaire et du secondaire. Un groupe de travail a développé un programme d'échanges trinationaux, TRISCHOLA, cofinancé par les fonds Interreg. Les incitations à la pratique des échanges scolaires à l'intérieur de l'espace frontalier n'ont cependant pas connu le succès espéré et beaucoup d'enseignants, pourtant volontaires, ont renoncé après une ou deux tentatives, faute de pouvoir gérer les difficultés d'ordre culturel de manière satisfaisante (Breugnot, 2007). II semble que les autorités n'aient pas prévu de prendre en compte, d'une part, les représentations et les convictions relatives aux critères de " normalité » de la relation adulte/enfants, enseignant/élèves dans un pays et dans l'autre et, d'autre part, aient fait abstraction des traumatismes familiaux, souvent tus mais pourtant présents dans nombre de familles alsaciennes. Les déchirements liés aux choix d'appartenance nationale et culturelle durant la dernière guerre mondiale ont laissé des cicatrices qui peuvent réapparaître en situation émotionnellement chargée et qui alourdissent les relations sans donner l'occasion de les verbaliser.

En revanche, des formes d'apprentissage précoce et intensif de «la langue du voisin » ont été mises en place avec un certain succès. Le nombre d'heures consacrées à l'apprentissage, allant de cinquante minutes réparties sur la semaine à douze heures hebdomadaires. Les rectorats de part et d'autre de la frontière se sont engagés à privilégier l'allemand et le français, et l'anglais n'est pratiquement pas enseigné dans les écoles primaires d'Alsace (moins de $5 \%$ des classes en comptant l'École européenne de Strasbourg) ni dans l'ouest du Bade-Wurtemberg. II reste enseigné dans le sud du Palatinat mais les classes bilingues français-allemand s'y sont également multipliées ces dernières années.

\footnotetext{
${ }^{4}$ http://www.conference-rhin-sup.org/ (consult. le $1^{\text {er }}$ déc. 2011).

${ }^{5}$ http://www.colingua.org (consult. le 11 nov. 2011).
} 
Ces choix de politiques éducatives ont été largement soutenus, voire motivés, par les petites et moyennes entreprises locales qui ont des besoins importants en personnel bilingue. En Alsace, presque la moitié des offres d'emploi comporte une exigence de connaissances, voire de maîtrise de l'allemand ${ }^{6}$, mais ces besoins économiques ne parviennent pas à convaincre réellement les parents et a fortiori les élèves de privilégier la « langue du voisin » par rapport à l'anglais. Et il semblerait que les autorités éducatives du Bade-Wurtemberg aient fini par céder, en 2011, à la pression des parents d'élèves pour rétablir l’offre de l'anglais.

Il est difficile de dire quelles seront les conséquences à moyen terme de ce déséquilibre qui ne manquera pas d'être exploité en Alsace où l'on vise pourtant à la généralisation de l'offre de l'enseignement précoce de la langue régionale et à son développement dans le second degré dans le cadre de la politique régionale des langues vivantes. Après de longues tergiversations, l'allemand est, aujourd'hui, considéré officiellement comme la forme écrite de la langue régionale et c'est à ce titre qu'ont été créées les classes bilingues. La création de nouveaux sites peut être proposée par l'Académie, les parents ou les municipalités. Le principe des classes bilingues repose sur l'enseignement d'une ou plusieurs matières dans la langue étrangère. Le nombre d'heures de cet apprentissage, dit en immersion, varie considérablement d'un côté et de l'autre de la frontière, allant de quatre heures hebdomadaires pour les enfants de six ans en Allemagne à douze heures dès la moyenne section de maternelle en Alsace. L'instauration de ces sites bilingues en Alsace dans le secteur de l'enseignement public doit beaucoup au militantisme des associations LEHRER (association professionnelle d'enseignants) et ABCM (Association pour le bilinguisme dès les classes maternelles) pour la sauvegarde de la langue régionale, mais le manque d'enseignants qualifiés perdure faute de compétences linguistiques suffisantes chez les candidats, et l'Inspection académique se voit contrainte de recruter des contractuels germanophones ne disposant pas toujours d'une grande expérience d'enseignement.

Comme nous l'avons évoqué plus haut, cette formule risque de se trouver fragilisée dans les années qui viennent, sans doute concurrencée par une demande d'anglais. Les parents s'étant mobilisés avec succès côté allemand pour la réintroduction de l'anglais à l'école primaire dans la bande frontalière, la demande d'une offre comparable ou du moins d'une introduction de l'anglais à l'école primaire s'exprime de plus en plus fort côté alsacien. Le choix de l'allemand en $6^{\mathrm{e}}$ en Alsace n'a pu être maintenu à $63 \%{ }^{7}$ que par l'instauration d'un système de classe " bilangues ", classes où sont proposés de manière équivalente et parallèlement l'anglais et l'allemand dès la 6 e .

\section{La réalisation des politiques de coopération au quotidien}

Comme le montre bien Yves Lacoste (2008), toute frontière engendre, même dans les contextes depuis longtemps pacifiés, un déséquilibre réel ou imaginé. Les stratégies adoptées par les populations vivant dans les espaces frontaliers sont en grande partie influencées par l'histoire et par les relations de hiérarchie économique. Même lorsque cette hiérarchie est faible, elle entraîne des relations de dominance. Celles-ci ne sont pas toujours conscientisées du côté des dominants, en revanche, elles sont toujours perçues du côté des dominés et gênent l'instauration d'une relation de confiance. Le déséquilibre transparaît entre autres dans les stratégies linguistiques adoptées dans ces espaces.

\section{Les pratiques linguistiques}

Les sections bilingues allemand-polonais créées dans l’Euregio Viadrina connaissent un fort déséquilibre, le nombre de Polonais apprenant l'allemand étant bien plus élevé que celui des Allemands apprenant le polonais - ces derniers ayant, d'ailleurs, pour la plupart, des attaches familiales en Pologne. Les études menées par les sociologues Schwarz et Jacobs (2004) montrent que si les relations entre étudiants allemands et étudiants polonais sont assez satisfaisantes pendant les cours, elles ne se poursuivent pratiquement pas en dehors de l'université, chaque nationalité retrouvant son cercle social national dans la vie privée.

Côté franco-espagnol, les efforts pour mettre en place une coopération hospitalière se sont heurtés à l'évolution, qui touche toute l'Espagne et n'a pas épargné l'espace frontalier, d'une raréfaction des locuteurs maîtrisant le

\footnotetext{
${ }^{6}$ http://www2.pole-emploi.fr/observatoire/publics/chomage_jeunes_portrait_statistique_18.html (consult. le 12 mai 2011 )

${ }^{7}$ Selon les chiffres de l'Inspection académique du Bas-Rhin (15 mai 2012).
} 
français (Loyer, 2006). II est encore fréquent qu'une partie des élèves n'apprennent qu'une langue étrangère et dans ce cas, c'est généralement l'anglais qui est proposé. Dans le contexte de crise économique que nous connaissons, nous assistons, alors que l'allemand a du mal à se faire reconnaître comme un " bon investissement " en Alsace, à un essor tout à fait considérable de la demande d'enseignement de l'allemand dans les lycées et universités espagnols. La baisse du nombre de locuteurs francophones dans l'espace frontalier franco-espagnol commence à poser problème pour certaines coopérations décidées alors que l'environnement linguistique était encore favorable au français. Côté français, l'espagnol est encore choisi à $75 \%$ comme seconde langue étrangère, mais ce chiffre s'explique en partie par le taux élevé de résidents espagnols ou d'origine espagnole. Ainsi, la coopération mise en place entre l'hôpital de San Sebastian et celui de Bayonne et qui prévoyait une flexibilité dans le recrutement des personnels et une mise en commun des réflexions stratégiques, souffre du manque de compétences en français côté espagnol que ne peuvent pallier les compétences en basque, d'autant plus que, comme nous l'avons évoqué plus haut, Bayonne n'est historiquement pas bascophone et qu'il est difficile de demander aux médecins de Bayonne d'apprendre l'euskara.

\section{Les stratégies parentales}

Dans l'espace franco-allemand, les positionnements observés sont multiples.

Côté français,

- une frange de la population, minoritaire mais très active milite pour la restauration d'un bilinguisme alsacien. Elle est à l'origine des associations LEHRER et ABCM. Cette dernière association s'est impliquée pour la création de classes financées en partie par le Conseil régional, en partie sur fonds privés. Les militants de ces associations visent en premier lieu un renouveau de l'identité régionale alsacienne, et seulement en second lieu une réelle communication transfrontalière. II s'agit pour eux de retrouver le bilinguisme alsacien perdu et considéré comme un élément fort de l'identité. Elles s'appuient sur les écrits d'universitaires militants tels que ceux de Jean Petit ou Claude Hagège ${ }^{8}$, qui soutiennent la thèse que les résultats obtenus dans l'ensemble des matières par les élèves de classes bilingues seraient supérieurs à ceux des classes monolingues du fait du bilinguisme. Les facteurs tels que l'investissement parental dans le suivi scolaire ou les attentes quant aux résultats escomptés ne semblent toutefois pas avoir été pris en compte. Ce dernier élément, cependant, pourrait sans doute expliquer, en partie du moins, les différences de résultats en langue entre les classes $\mathrm{ABCM}$ et les classes du secteur public.

- Une faible partie considère que, compte tenu de la proximité, il serait dommage de ne pas parler la langue de l'autre, surtout dans le cadre d'une Europe unie souhaiterait une plus grande ouverture. Ils témoignent d'une curiosité à l'égard de la culture allemande ou suisse et considèrent les projets communs avec bienveillance.

- Une grande partie de la population, enfin, vit adossée à la frontière, ignorant plus ou moins le monde existant de l'autre côté. Les rares occasions de passer la frontière sont l'achat de nourriture ou de produits ménagers, moins chers en Allemagne, et la fréquentation de certaines infrastructures telles que piscines ou saunas, jugées plus luxueuses.

Les parents d'élèves qui choisissent un enseignement intensif ou une classe bilingue peuvent appartenir à ces trois catégories, allant des militants régionaux aux indifférents en passant par les " européanistes ». Ceux qui tendent à ignorer l'existence du voisin allemand et qui ont fait le choix du bilinguisme " allemand " l'ont souvent fait comme on faisait autrefois le choix de la filière " allemand-latin » en classe de sixième. Les classes d'immersion ne forment pas, à proprement parler, des classes d'élite car il n'y a pas de niveau exigé officiellement, mais les parents non diplômés, non familiers du système scolaire ou dont les enfants ont des difficultés ne font pas la démarche de demander leur inscription dans ces classes. Or cela permet de laisser les enfants dans l'école publique du quartier, de respecter la carte scolaire, d'éviter de longs trajets ou de devoir opter pour une école privée tout en profitant d'une mixité sociale plus faible.

\footnotetext{
${ }^{8}$ Jean Petit (1921-2003), psycholinguiste, professeur honoraire de I'université de Reims, membre du conseil scientifique de I'ISLR; Claude Hagège, linguiste, professeur au Collège de France.
} 
Cependant, ces motivations sont souvent jugées inavouables par les parents et le message transmis aux enfants s'en trouve distordu. Mis en relation avec une image du pays ou de la langue cible peu reconnue, le manque de congruence peut expliquer les résultats mitigés obtenus en langue, les enfants ayant du mal à mettre un sens derrière cet apprentissage. Il suffit alors quelquefois qu'un enfant exprime le message parental non formulé pour que la critique se répande dans tout le groupe classe et qu'émerge une sorte de mépris à l'égard de la langue et de la culture enseignée (Breugnot, 2007).

La confusion est engendrée aussi par un discours et un comportement institutionnel contradictoires. Officiellement, les classes d'immersion donneraient donc de meilleurs résultats, non seulement dans la langue étrangère mais également dans les autres matières comme la langue maternelle ou les mathématiques alors que, partout, on découragera les parents d'élèves "faibles » d'inscrire leur enfant dans le cursus bilingue et qu'on sortira les élèves en difficulté des classes qu'ils fréquentent déjà. On observe ainsi de bons résultats scolaires dans les matières classiques mais un niveau en langue assez médiocre au regard du temps investi.

Côté allemand, les parents qui optent pour l'enseignement bilingue répondent à des soucis comparables de stratégie scolaire. La compréhension de l'approche immersive varie cependant car les efforts demandés aux élèves du primaire de part et d'autre de la frontière ne sont guère comparables. Pour citer un exemple réel et moins caricatural qu'il n'y paraît : une enseignante de Strasbourg donnait une dizaine de mots allemands à apprendre chaque jour tandis qu'un enseignant de Landau fut contacté par des parents qui craignaient de dégoûter leurs enfants de l'effort pour cinq mots français à apprendre dans la semaine. Les efforts et contraintes imposés aux enfants reposent sur une conception du temps et de l'enfance différente de part et d'autre de la frontière (Breugnot, 2010).

La classification à l'intérieur de la population est moins aisée, plusieurs sentiments cohabitant fréquemment chez les mêmes personnes. Le choix d'inscrire son enfant dans un cursus bilingue allemand-français est un choix par défaut pour une grande partie des parents, qui opteraient pour un bilinguisme allemand-anglais si la possibilité s'offrait (Laabs, 2009). La préservation d'une proximité culturelle et la construction d'un bilinguisme régional les concernent peu. Soit ils souhaitent voir dans le passage de frontière un départ vers un lieu de villégiature, l'occasion de se procurer des produits alimentaires encore rares en Allemagne ou bien auxquels ils souhaitent garder un caractère authentiquement exotique, et, dans ces deux cas, c'est davantage une affirmation claire des différences qui est recherchée: soit ils voient dans l'Alsace un prolongement naturel de l'Allemagne, dont le rattachement politique à la France n'est dû qu'aux aléas de l'Histoire mais dont le bilinguisme est un phénomène naturel et qui pour cette raison perdure ${ }^{9}$.

L'enfance et l'éducation sont sans doute les éléments de notre identité les plus difficiles à mettre objectivement en perspective. Les souvenirs que nous en gardons et les valeurs qui nous ont été transmises échappent aux prises d'une relativisation intellectuelle.

Les deux exemples qui suivent sont significatifs de l'importance que garde notre expérience d'un système scolaire et de l'impact qu'elle peut avoir sur nos décisions.

Une partie, certes peu significative quantitativement mais pourtant révélatrice, des résidents de nationalité allemande ou de couple mixtes, côté français, s'est organisée pour assurer la scolarisation de leurs enfants dans le système allemand. Ainsi existent un système de co-voiturage entre Strasbourg et Kehl ou Offenburg et une organisation par roulement pour assurer la prise en charge des enfants l'après-midi. Pour la majorité d'entre eux, il ne s'agit pas de préparer un « retour au pays » mais de privilégier un système qui leur semble plus performant ou mieux adapté, plus respectueux des personnalités enfantines (Gaiger-Jaillet, 2005).

\footnotetext{
${ }^{9}$ Selon les sources, les chiffres concernant les locuteurs dialectophones en Alsace vont de $4 \%$ de dialectophones réceptifs à plus de $60 \%$ de locuteurs pratiquants. Les résultats sont manifestement influencés par un positionnement idéologique ou par une définition différente de la dialectophonie.
} 
Comme nous l'avons évoqué plus haut, les échanges scolaires, malgré un encouragement constant au niveau des rectorats, restent rares et souvent ne perdurent pas au-delà de deux ans (Breugnot, 2008). Les différences de culture scolaire concernant la discipline, le déroulement des activités et les principes théoriques expliquent en partie ces difficultés, les enseignants ayant beaucoup de mal à relativiser les valeurs qu'ils rattachent à l'école.

En outre, la proximité ne favorise pas forcément la tolérance. Elle ne suscite pas non plus la curiosité et il reste plus facile de motiver des adolescents de Lyon à se rendre à Berlin et inversement que de convaincre des élèves de Strasbourg ou d'Offenburg qu'il peut valoir la peine de franchir le Rhin. L'attrait de ces échanges repose pour beaucoup sur les charmes de l'exotisme et peu d'enseignants ont, jusqu'à présent, su trouver un ersatz.

\section{Les principaux freins à l'établissement d'un vrai débat}

Les motivations pour développer des projets transfrontaliers peuvent d'être d'ordre économique, politique, idéaliste, identitaire... Elles ont en commun d'être radicalement tournées vers l'avenir, d'avoir des objectifs ambitieux sans toujours mesurer l'importance et la complexité des conditions de réalisation.

Les initiateurs de projets d'ordre économique aimeraient voir les problèmes linguistiques, qu'ils identifient généralement comme principal handicap à la coopération, pris sérieusement en charge par les institutions. Ces dernières y répondent de leur mieux mais se voient limitées dans leurs actions par l'interdit qui pèse sur la parole. En effet, une grande part des personnes impliquées dans ces projets ont des convictions idéologiques fortes qui fonctionnent quelquefois comme des croyances, et il devient alors difficile d'établir un débat car toute remise en cause, si limitée soit-elle, est perçue comme une agression et la personne qui la formule comme un adversaire ${ }^{10}$.

Il en va de même pour les sentiments engendrés par les dominances économiques. Même si celles-ci tendent à s'aplanir, on peut encore observer une assurance, une affirmation de soi, souvent inconsciente chez les populations au pouvoir d'achat le plus élevé et une mauvaise perception, ou un déni, de l'effet produit par cette assurance sur les populations « moins riches ».

Le manque de congruence entre les politiques, les motivations affichées et les motivations réelles, le flou qui persiste autour des véritables enjeux sans qu'il soit possible d'aborder la question ouvertement, empêche l'instauration d'une réflexion apaisée qui serait mieux apte à prendre en compte les résistances.

L'hypothèse émise par le sociologue Jean Viard, en 1997, d'une "demande locale de France " en Alsace mériterait peut-être d'être revisitée. Pour lui, les votes Front National, inexplicables avec les arguments habituels du chômage, du fort taux d'immigrés et d'un faible niveau de revenu, l'Alsace comptant parmi les régions les plus privilégiées de France, s'expliqueraient par une demande ambiguë d’un lien plus fort à l'État national qui n'exercerait plus sa fonction protectrice face à l'ouverture des frontières. Les populations seraient confrontées à une angoisse diffuse de se voir absorbées par l'étranger. La même explication vaudrait également pour la région Provence-Alpes-Côte-d'Azur, qui se verrait rattachée malgré elle à une entité méditerranéenne. C'est comme si le sentiment d'appartenance avait besoin de l'exclusion pour s'épanouir, comme si les fraternités tribales prévalaient sur la fraternité générale.

Ainsi, le décalage entre le positionnement très idéaliste, très impliqué mais minoritaire des tenants d'une régionalisation transfrontalière et le reste de la population, peu concernée par la question, cache des résistances plus ou moins inconscientes qui ne contribuent pas à une évolution harmonieuse.

\section{Bilan et perspectives}

La construction des espaces frontaliers constitue un vaste champ d'études. Toute tentation de simplification entraîne vite vers une vision partielle et faussée de la réalité. Les intérêts y sont de nature multiple. Le bon sens, une certaine évidence, voudrait qu'à l'intérieur d'une Europe que chacun souhaite unie, les frontières développent une porosité qui les ferait à terme, disparaître.

\footnotetext{
${ }^{10}$ Pour cette raison, quelques collègues-informateurs n'ont pas souhaité être cités.
} 
Lorsque l'on cherche à comprendre les phénomènes en jeu dans l'évolution de la notion de frontière à l'intérieur d'une entité englobant plusieurs nations comme l'Europe, on voit tout d'abord les nombreuses réalisations techniques et économiques mises en place au niveau transfrontalier. Ces réalisations créent des besoins en interaction et donc en aptitude à communiquer. L'une des réponses apportées spontanément a donc été de développer des compétences linguistiques partagées.

La stratégie politique visait à créer un sentiment d'appartenance géographique partagé en créant des régions transfrontalières, qui se transformeraient peu à peu en identité partagée et effacerait ainsi la frontière comme barrière.

La logique de ces réflexions s'est heurtée à la complexité de la psychologie humaine et la plupart des études menées dans ces espaces montrent aujourd'hui que la proximité ne simplifie pas nécessairement la communication. Elle entraîne des cristallisations émotionnelles résultant, d'une part, des marques laissées par I'histoire dans les cultures familiales, et d'autre part, de la nécessité de protéger une identité.

Développer un sentiment d'appartenance à une nouvelle entité géographique est un processus long, dont on ne peut même pas être certain qu'il soit souhaitable et surtout qui ne se décrète pas. Les évolutions actuelles de la cohabitation et de la coopération européennes montrent assez la distance entre discours, représentations et même honnête volonté politique et la fragilité des solidarités face aux épreuves.

Les relations établies, souvent péniblement construites, dans les espaces frontaliers sont-elles de nature à résister et à répondre aux tensions et aux sentiments de rejet qui s'expriment au niveau des nations ?

II nous semble que deux axes d'intervention puissent encore être activés, l'un au niveau macro par l'expression d'une réelle volonté politique d'unification, l'autre au niveau micro qui se traduirait par une réelle écoute des besoins et désirs des habitants.

\section{Références bibliographiques}

Bothorel-Witz, A. (2000). Les langues en Alsace. DiversCité Langues [En ligne]. http://www.teluq.uquebec.ca/diverscite [consult. le 9 déc. 2011]

Berger, C. (2007). Explorer les frontières. L'initiation à la recherche anthropologique comme outils de formation. Dans J. Breugnot (dir.), Les espaces frontaliers (p. 163-174). Berne : Peter Lang.

Breugnot, J. (2007). Rencontres scolaires en zone frontalière : l'expression des émotions dans la prévention des conflits. Dans J. Breugnot (dir.), Les espaces frontaliers (p. 175-186). Berne : Peter Lang.

Breugnot, J. (2008). La formation interculturelle des enseignants en zone frontalière. Dans V. Marie et N. Lucas (dir.), Regards sans frontières sur la formation des enseignants (p. 173-187). Paris : Éditions Le Manuscrit.

Breugnot, J. (2010). L'école élémentaire en France et en Allemagne. Dans : Glossaire pour l'École maternelle et élémentaire/Glossar für die Grundschule (p. 20-27). Berlin : Office franco-allemand pour la jeunesse.

Breugnot J. (2011). La place du contexte dans l'approche immersive. Beiträge zur Fremdsprachenvermittlung, 50, 67-73. http://www.vep-landau.de/bzf/2011_50/bzf_Heft_50-2011.htm

Fouquet, F. (2007). Dis-moi les couleurs du monde! Dans J. Breugnot (dir.), Les espaces frontaliers (p.149-162). Berne : Peter Lang.

Gaiger-Jaillet, A. (2005). Flux transfrontaliers scolaires au cœur l'Europe: va-t-on vers des bassins scolaires transfrontaliers?, dans C. Van den Avenne (éd.), Mobilités et contacts de langues (p. 311-329). Paris : L'Harmattan (Espaces discursifs) [Actes du $3^{\mathrm{e}}$ colloque du Réseau français de sociolinguistique (RSF), 2021 mars 2003, à l'ENS Lyon]. 
Huck, D. (2005). L'enseignement de l'allemand à l'école élémentaire en Alsace. Questions de méthode : les manuels en usage entre 1952 et 1990. Histoire de l'éducation, 106, 217-267. http://histoireeducation.revues.org/1085

Kurcz Z., (2006). Wirtschaftliche und soziokulturelle Probleme der polnisch-deutschen Grenzregion. Dans J. Breugnot et M.Molz (dir.), Europa konkret! Grenzräume als Chance für Bildungsinnovationen (p. 23-38). Frankfurt am Main : Knecht Verlag.

Kurcz, Z. (2007). Problèmes socioculturels et économiques dans la zone frontalière germano-polonaise. Dans J. Breugnot, Les espaces frontaliers : laboratoires de la citoyenneté européenne (p. 9-23). Berne : Peter Lang.

Laabs, F. (2009). Immersive Didaktik (Wissenschaftliche Prüfungsarbeit [mémoire de fin d'étudess]). Universität Koblenz-Landau.

Lacoste, Y. (dir.) (1993). Dictionnaire de géopolitique. Paris : Flammarion.

Lacoste, Y. (2008). La longue histoire d'aujourd'hui. Paris : Larousse.

Loyer, B. (2006). Géopolitique d'une frontière : I'exemple franco-espagnol dans les Pyrénées atlantiques, dans J. Breugnot (dir.), Les espaces frontaliers : laboratoires de la citoyenneté européenne (p. 25-40). Peter Lang.

Odgers, O. (2001). Identités frontalières : immigrés mexicains aux États-Unis. Paris : L'Harmattan.

Schwarz, A. et Jacobs, J. (2004). Bangen an der Oder - Ängste und Hoffnungen spiegeln sich. Osteuropa, 54(5-6), Sonderheft zur EU-Osterweiterung: Die Einigung Europas - Zugkraft und Kraftakt, mai-juin.

Viard, J. (1997). Une demande locale de France. Dans B. Remaux et P. Breton (dir.), L'Appel de Strasbourg. Strasbourg : La Nuée bleue. 